\title{
Athletic Performance Prediction Model Design Based on Grey BP Neural Network
}

\author{
JIA Ling ${ }^{1}$, WANG Qing-bin ${ }^{1}$ \\ 1.Zhuhai College of Jilin University, Zhuhai Guangdong, 519041 China
}

\begin{abstract}
Keywords: Athletic Performance; Women's Heptathlon; Grey BP Neural Network Prediction Model
\end{abstract}
\begin{abstract}
The best annual performances of the world women's pentathlons during 2005 2013 are statistically collected in this article, and the prediction of the best performance of the world women's heptathlon in 2013 is taken as the research object. According to the best annual performances of the world women's heptathlons during 2005 2012, the athletic performance prediction model composed of GM(1,1) grey prediction model and BP neural network prediction model in serial connection is established in this article, and this model is applied to predict the best annual performance of the world women's heptathlon in 2013. Through the comparison of the actual value of the best annual performance of the world women's heptathlon in 2013 and the predicted value of the model, the application of the grey BP neural network prediction model in athletic performance prediction is researched and analyzed in this article. The research result shows that for the athletic performance prediction problem, the grey BP neural network prediction model has the features of high prediction accuracy, simple application and strong generalization performance, and this model is also superior to single $\mathrm{GM}(1,1)$ grey prediction model and BP neural network model.
\end{abstract}

\section{Introduction}

With significant practical value, athletic performance prediction is favorable not only for providing training objectives to the athletes, but also for providing reference to the research on the development of athletic sports. The traditional prediction methods mainly include time series method, analogy, regression analysis method, etc. The above predictions are mainly based on mass analyzable data, and the research scope is usually limited to static problems. Specifically, the athletic performance prediction problem has the features of less analyzable data, strong data randomness, many influencing factors, mutual influence and complex factor relationship, so the result of the athletic performance prediction, obtained by traditional prediction methods, is not ideal.

\section{Introduction to Grey BP Neural Network Model}

Composed of GM(1,1) grey prediction model and BP neural network prediction model in serial connection, the grey BP neural network model established in this article not only has the functions possessed by the grey prediction model for processing small samples and poor information, but also has the features of robustness, fault tolerance, etc. of BP neural network model, thus comprehensively presenting the interdisciplinary property of the grey information mining.

Among the artificial neural network models, BP neural network model is a neural network model which is most thoroughly researched by people. According to relevant statistical information, the application of BP neural network model occupies $80 \% \sim 90 \%$ of the applications of artificial neural network models. In detail, BP neural network model has a three-layer neural network structure, namely: input layer, hidden layer and output layer, wherein the layers are fully connected with each other and the connection weight values are repeatedly learned and trained through error back propagation according to the training patterns till the corresponding weight value can meet the requirements of the training patterns. Moreover, BP neural network model can approximate to any nonlinear function through trainings already provided with training patterns and can rapidly and 
accurately process the problems of the nonlinear systems, thus being widely applied in the fields of function approximation, prediction, system identification, classification, data compression, etc.

BP neural network model is established through the following four steps:

(1) Research problem, extract elements and collect training patterns;

(2) Set the parameters of BP neural network according to the problem;

(3) Establish training samples, input the parameters in the training samples into BP neural network system established thereby and compare the system output with the expected pattern; if any error, please execute step (4); or else, please return to step (3);

(4) Correct the connection weight values of various layers of the system through back propagation.

\section{Establishment of Grey BP Neural Network Prediction Model}

The seven single sports of women's heptathlon are mutually related and various athletic performances can influence each other, so it is necessary to consider the relationship among these sports during the performance prediction process. In the grey BP neural network prediction model established in this article, BP neural network model aims at comprehensively researching the mutual relationship among the athletic performances while $\mathrm{GM}(1,1)$ grey prediction model aims at making full use of the time series of the statistical data in order to mine the temporal relationship among the data.

\section{Statistical Information of the Best Annual Performances of Women'S Heptathlon}

As women's comprehensive sport event, women's heptathlon composed of 100m hurdles, high jump, shot, 200m run, long jump, javelin and 800m run involves run, jump and shoot sports and has high requirements for the physical quality, the psychological quality, the technical merit, etc. of the athletes. The best annual performances of the world women's heptathlons during 2003 2013 are statistically collected in this article, and the statistical performances are as shown in Table 1.

Table 1 The Best Annual Performances of the World Women's Heptathlons during 2003 2013

\begin{tabular}{cccccccc}
\hline & 100m Hurdles & High Jump & Shot & 200m Run & Long Jump & Javelin & 800m Run \\
\hline 2003 & 13.18 & 1.94 & 14.19 & 22.98 & 6.68 & 49.90 & 132.12 \\
2004 & 13.21 & 1.91 & 14.77 & 23.27 & 6.78 & 48.89 & 134.15 \\
2005 & 12.62 & 1.91 & 12.61 & 24.12 & 6.78 & 53.07 & 134.66 \\
2006 & 13.35 & 1.89 & 14.56 & 23.86 & 6.65 & 46.94 & 134.95 \\
2007 & 13.15 & 1.95 & 14.81 & 23.38 & 6.85 & 47.98 & 132.98 \\
2008 & 13.44 & 1.80 & 17.29 & 24.39 & 6.63 & 48.60 & 137.72 \\
2009 & 12.93 & 1.92 & 14.14 & 23.25 & 6.29 & 43.54 & 132.22 \\
2010 & 12.95 & 1.89 & 14.05 & 23.21 & 6.43 & 46.71 & 130.18 \\
2011 & 13.32 & 1.83 & 14.17 & 23.50 & 6.61 & 52.95 & 128.04 \\
2012 & 12.54 & 1.86 & 14.28 & 22.83 & 6.48 & 47.49 & 128.65 \\
2013 & 13.63 & 1.86 & 13.92 & 23.93 & 6.48 & 47.90 & 131.58 \\
\hline
\end{tabular}

Original Data Processing

Before grey BP neural network prediction model learning and training, it is necessary to implement the normalization processing operations for the total performances and the single-sport performances $f$ the women's heptathlons in order to make all sample values in the interval of $[0,1]$. Afterwards, the maximum values of the single-sport performances among the best annual performances of the women's heptathlons during 2003 2013 are taken as the denominator, and the performances of the corresponding sports in other years are taken as the numerator. The data processed as above are shown in Table 2. 
Table 2 Normalization Data of the Best Annual Performances of the World Women's Heptathlons during 2003 2013

\begin{tabular}{cccccccc}
\hline & 100m Hurdles & High Jump & Shot & 200m Run & Long Jump & Javelin & $800 \mathrm{~m}$ Run \\
\hline 2003 & 0.9670 & 0.9949 & 0.8207 & 0.9422 & 0.9752 & 0.9403 & 0.9593 \\
2004 & 0.9692 & 0.9795 & 0.8587 & 0.9541 & 0.9898 & 0.9212 & 0.9741 \\
2005 & 0.9259 & 0.9795 & 0.7293 & 0.9889 & 0.9898 & 1.0000 & 0.9778 \\
2006 & 0.9795 & 0.9692 & 0.8421 & 0.9783 & 0.9708 & 0.8845 & 0.9799 \\
2007 & 0.9648 & 1.0000 & 0.8566 & 0.9586 & 1.0000 & 0.9041 & 0.9656 \\
2008 & 0.9861 & 0.9231 & 1.0000 & 1.0000 & 0.9679 & 0.9158 & 1.0000 \\
2009 & 0.9486 & 0.9846 & 0.8178 & 0.9533 & 0.9182 & 0.8204 & 0.9601 \\
2010 & 0.9501 & 0.9692 & 0.8126 & 0.9516 & 0.9387 & 0.8802 & 0.9453 \\
2011 & 0.9773 & 0.9385 & 0.8195 & 0.9635 & 0.9650 & 0.9977 & 0.9300 \\
2012 & 0.9200 & 0.9538 & 0.8259 & 0.9360 & 0.9460 & 0.8949 & 0.9341 \\
2013 & 1.0000 & 0.9538 & 0.8051 & 0.9811 & 0.9460 & 0.9026 & 0.9554 \\
\hline
\end{tabular}

In this article, the dimension-fixed and recursion-compensated $\operatorname{GM}(1,1)$ grey model is established on the basis of the statistical single-sport performances as follows: firstly, establish GM(1,1) grey model according to the single-sport data during 2003 2006 and predict the normalization data corresponding to the performances in 2007; then, adopt the data during 2004 2007 to predict the normalization data corresponding to the performances in 2008; then, obtain the predicted values of the normalization data corresponding to the performances during 2007 2011. The predicted data obtained as above are shown in Table 3.

Table 3 Predicted Data of the Best Annual Performances of the World Women's Heptathlons during 2007 2013

\begin{tabular}{cccccccc}
\hline & 100m Hurdles & High Jump & Shot & 200m Run & Long Jump & Javelin & 800m Run \\
\hline 2007 & 0.9687 & 0.9658 & 0.7958 & 0.9980 & 0.9647 & 0.9003 & 0.9831 \\
2008 & 0.9958 & 1.0037 & 0.9419 & 0.9454 & 0.9972 & 0.8353 & 0.9623 \\
2009 & 0.9835 & 0.9197 & 1.0728 & 1.0011 & 0.9767 & 0.9331 & 1.0023 \\
2010 & 0.9506 & 0.9536 & 0.8555 & 0.9654 & 0.8832 & 0.8011 & 0.9698 \\
2011 & 0.9259 & 1.0053 & 0.6994 & 0.9205 & 0.9124 & 0.8361 & 0.9148 \\
2012 & 0.9878 & 0.9190 & 0.8183 & 0.9664 & 0.9884 & 1.0928 & 0.9154 \\
2013 & 0.9198 & 0.9384 & 0.8372 & 0.9350 & 0.9572 & 0.9385 & 0.9253 \\
\hline
\end{tabular}

Establishment of Training Samples

In the grey BP neural network prediction model established in this article, the predicted values of the seven single-sport athletic performances of the women's heptathlon are taken as the input of $\operatorname{GM}(1,1)$ grey prediction model and the actual performances are taken as the output. The training samples include seven samples, the predicted data of the grey prediction models for 2007 2011 and the actual data are respectively taken as the input and the output. The input matrix and the output matrix are respectively set as $\mathrm{P}$ and $\mathrm{T}$ : 


$$
\begin{aligned}
P & =\left[\begin{array}{lllllll}
0.9687 & 0.9658 & 0.7958 & 0.9980 & 0.9647 & 0.9003 & 0.9831 \\
0.9958 & 1.0037 & 0.9419 & 0.9454 & 0.9972 & 0.8353 & 0.9623 \\
0.9835 & 0.9197 & 1.0728 & 1.0011 & 0.9767 & 0.9331 & 1.0023 \\
0.9506 & 0.9536 & 0.8555 & 0.9654 & 0.8832 & 0.8011 & 0.9698 \\
0.9259 & 1.0053 & 0.6994 & 0.9205 & 0.9124 & 0.8361 & 0.9148
\end{array}\right]^{T} \\
T & =\left[\begin{array}{lllllll}
0.9648 & 1.0000 & 0.8566 & 0.9586 & 1.0000 & 0.9401 & 0.9656 \\
0.9861 & 0.9231 & 1.0000 & 1.0000 & 0.9679 & 0.9158 & 1.0000 \\
0.9486 & 0.9846 & 0.8178 & 0.9533 & 0.9182 & 0.8204 & 0.9601 \\
0.9501 & 0.9692 & 0.8126 & 0.9516 & 0.9387 & 0.8802 & 0.9453 \\
0.9773 & 0.9385 & 0.8195 & 0.9635 & 0.9650 & 0.9977 & 0.9300
\end{array}\right]^{T}
\end{aligned}
$$

\section{Establishment of BP neural Network Prediction Model}

The neural network prediction model established in this article includes one hidden layer. As mentioned above, the neural network includes 7 inputs and 7 outputs, namely: the input layer and the output layer respectively include 7 nerve cells. Additionally, the number of the nerve cells of the hidden layer can be researched according to the following empirical formula:

$$
i=\sqrt{n+m}+a
$$

In the above formula, $n$ is the number of the nerve cells of the input layer, $m$ is the number of the nerve cells of the output layer, and $a$ is a value between 0 and 1 . According to the above empirical formula, the number of the nerve cells of the hidden layer is 7.

BP neural network model includes many types of transfer functions, training functions, learning functions and performance functions. For example, the transfer functions include $\mathrm{S}$ logarithmic function, S tangent function, pure linear function, etc.; the training functions include BFGS Quasi-Newton BP algorithm function, gradient BP descent algorithm function, gradient descent momentum BP algorithm function, etc.; the learning functions include gradient descent weight learning function, gradient descent momentum weight learning function, etc.; the performance functions include mean square error performance function, mean square error normalization performance function, etc. In this article, S tangent transfer function and pure linear function, gradient descent momentum BP algorithm function, gradient descent momentum weight learning function and mean square error normalization function are selected for BP neural network model. Meanwhile, for the BP neural network model, the maximum training frequency is 10,000 times, the training accuracy is 0.005 and the training display interval is 500 times.

\section{Conclusion}

For such comprehensive athletic sports as women's heptathlon, the sports included therein can influence each other and the athletic performances thereof also have certain mapping relationship. In order to better predict the single-sport performances, it is necessary to make full use of the known data.

The grey BP neural network prediction model not only has the features of robustness, fault tolerance, etc. of BP neural network prediction model, but also makes full use of $\mathrm{GM}(1,1)$ grey prediction model to mine the temporal relationship among the data. For the multiple-factor prediction problems, the prediction accuracy of this model is not inferior to that of $\mathrm{GM}(1,1)$ grey prediction model.

\section{Acknowledgement}

The zhuhai college of jilin university, one hundred engineering project funds 


\section{References}

[1] Lv Z, Tek A, Da Silva F, et al. Game on, science-how video game technology may help biologists tackle visualization challenges[J]. PloS one, 2013, 8(3): 57990.

[2] Su T, Wang W, Lv Z, et al. Rapid Delaunay triangulation for randomly distributed point cloud data using adaptive Hilbert curve[J]. Computers \& Graphics, 2016, 54: 65-74.

[3] Lin Y, Yang J, Lv Z, et al. A Self-Assessment Stereo Capture Model Applicable to the Internet of Things[J]. Sensors, 2015, 15(8): 20925-20944.

[4] Yang J, Chen B, Zhou J, et al. A Low-Power and Portable Biomedical Device for Respiratory Monitoring with a Stable Power Source[J]. Sensors, 2015, 15(8): 19618-19632. 